\title{
Formation of Hydrogen Radical in Hydrogen Nanobubble Water and its Effect on Copper Toxicity in Chlorella
}

Shu Liu ${ }^{1,2}$, Jiayao Li ${ }^{1}$, Seiichi Oshita ${ }^{3}$, Mohammed Kamruzzaman ${ }^{4}$, Minming Cui $^{5}$, Wenhong Fan ${ }^{1,2, *}$

${ }^{1}$ Department of Environmental Science and Engineering, School of Space and Environment, Beihang University, Beijing 10191, China

${ }^{2}$ Beijing Advanced Innovation Center for Big Data-Based Precision Medicine, Beihang University, Beijing, 10191, China

${ }^{3}$ Graduate School of Agricultural \& Life Sciences, The University of Tokyo, Yayoi 11-1, Bunkyo-ku, Tokyo 113-8657, Japan

${ }^{4}$ Department of Agricultural and Biological Engineering, University of Illinois at Urbana-Champaign, 1304 West Pennsylvania Avenue, Urbana, IL 61801, USA.

${ }^{5}$ Department of Earth and Planetary Sciences, Johns Hopkins University, Baltimore, MD 21218-2687, USA

* Corresponding author: 
Wenhong Fan, E-mail: fanwh@,buaa.edu.cn

TEL: $010-61716810$

FAX: 010-61716810

Number of figures and tables: 4 figures 1 tables

Number of pages: 6 pages

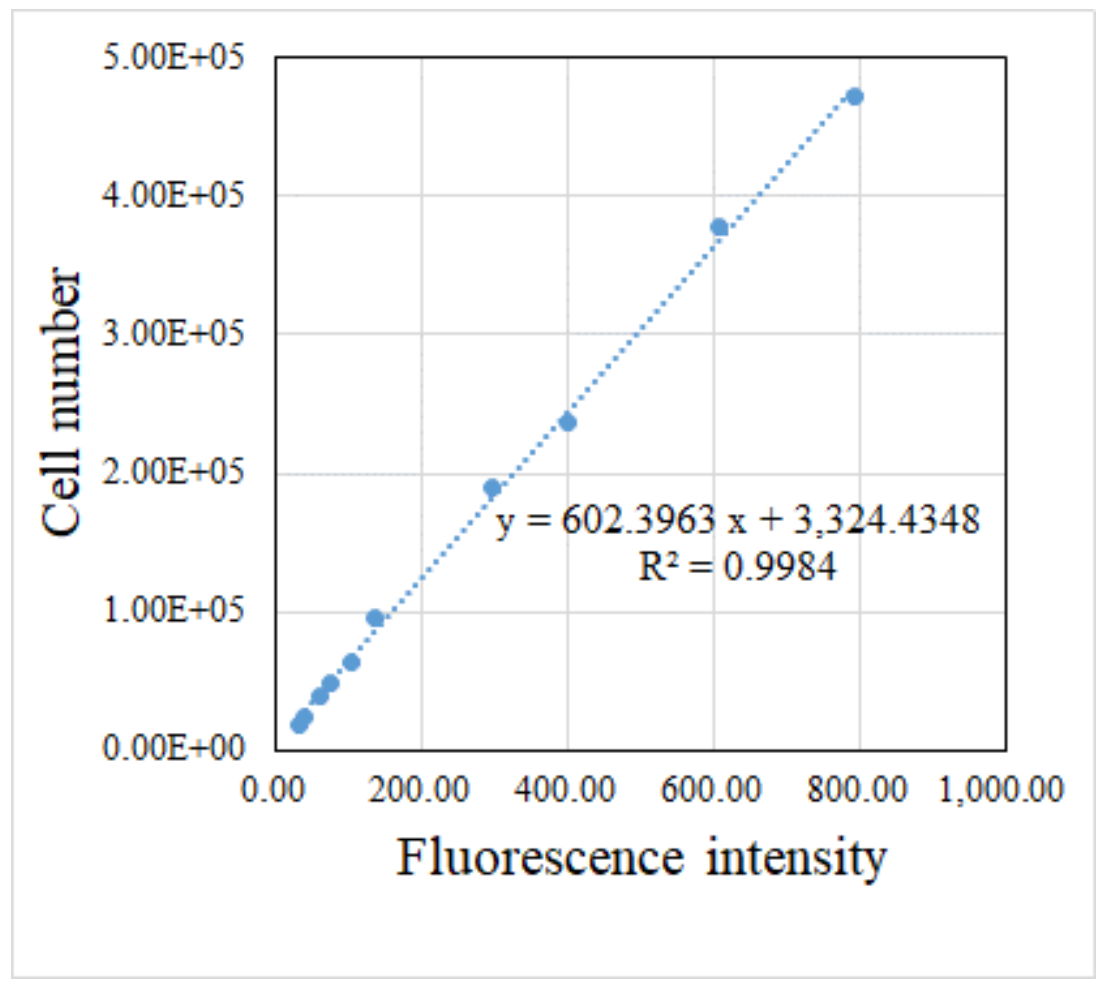

Figure S1 Calibration curves for green algae Chlorella grown in BG11 media based on fluorescence excitation of chlorophyll. 

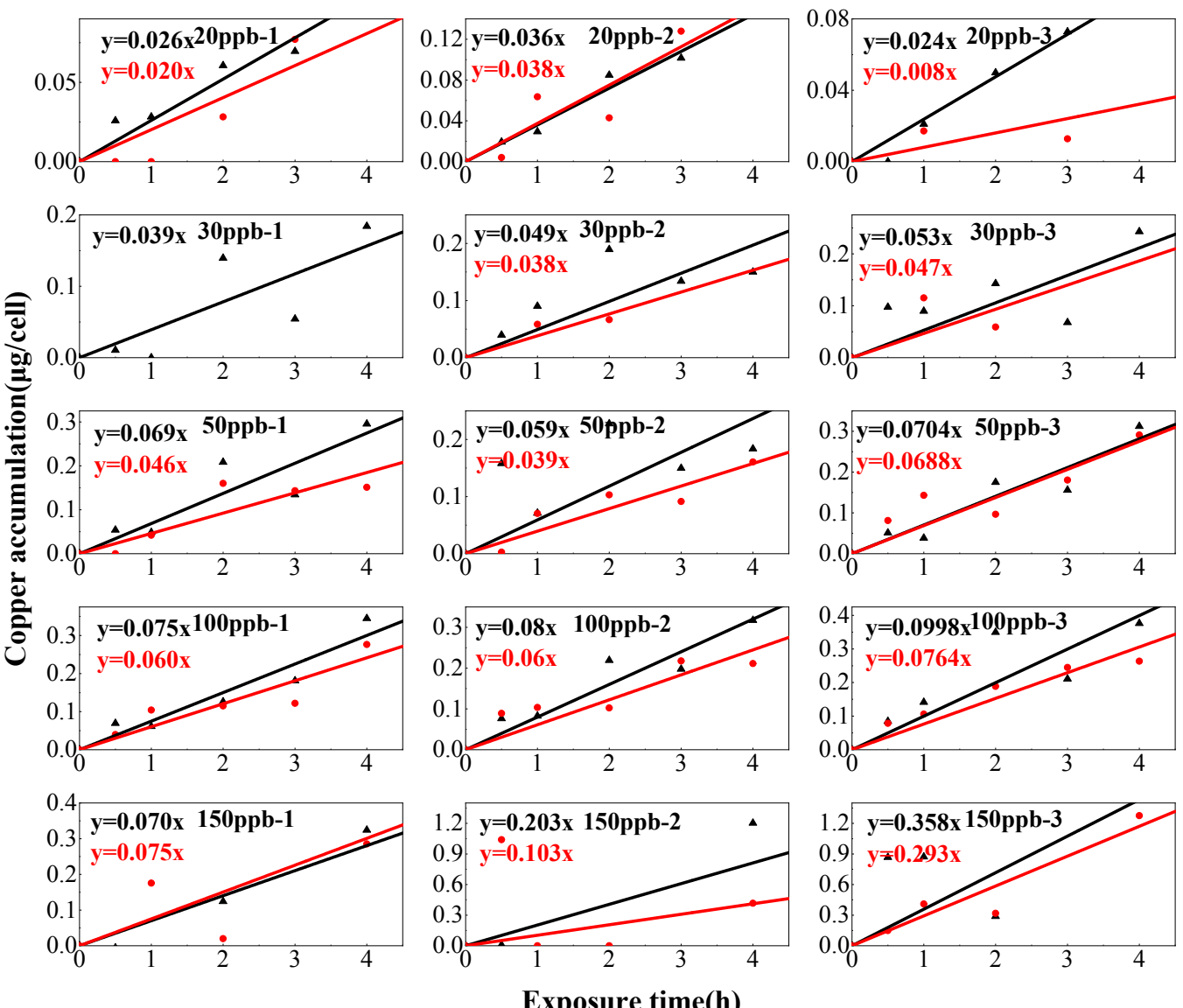

Exposure time(h)

Figure S2. Changes in intracellular $\mathrm{Cu}$ contents with exposure time at various

$\mathrm{Cu}$ concentrations $(20,30,50,100$, and $150 \mathrm{ppb})$ in the short-term uptake experiments for Chlorella in hydrogen NB and control waters. 


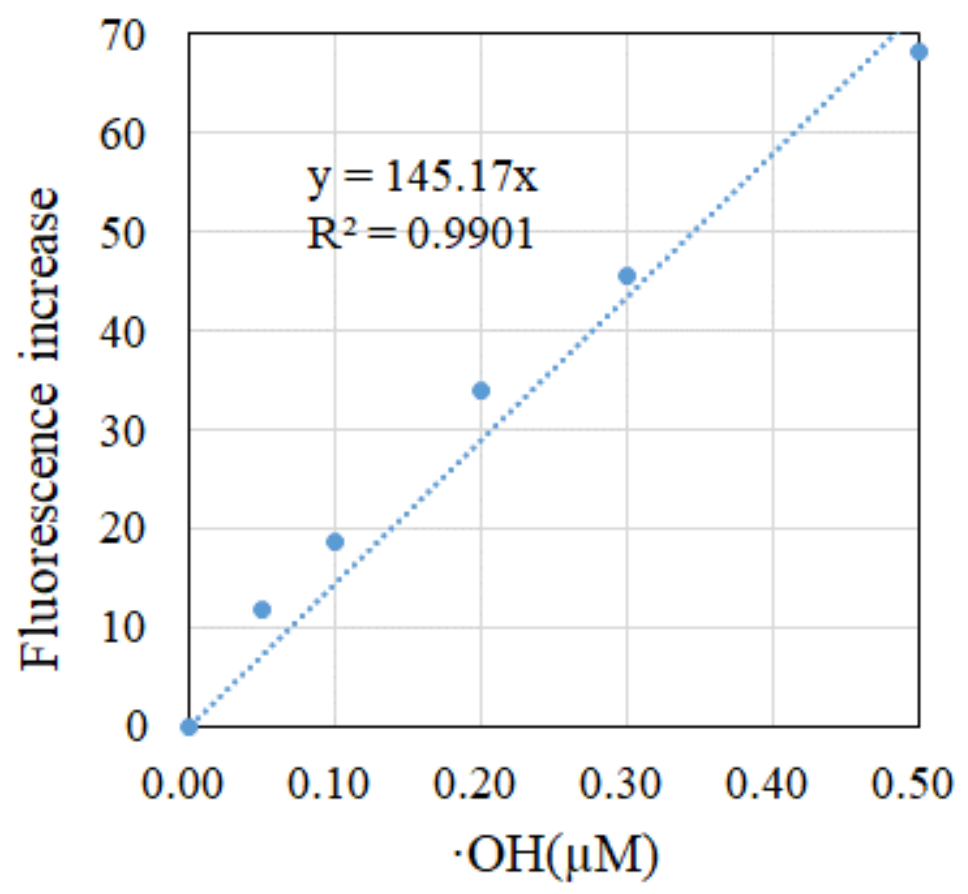

Figure S3 Fluorescence response of APF (0.1 M, pH 7.4) to $\cdot \mathrm{OH}$. The fluorescence intensity was determined at $515 \mathrm{~nm}$ with excitation at $490 \mathrm{~nm}$. Detection of $\cdot \mathrm{OH}$ in the Fenton reaction (In the horizontal axis, the concentration of Ferrous iron (II) is equal to that of hydroxyl radicals, $\mathrm{H}_{2} \mathrm{O}_{2}(0.1 \mathrm{mM})$ was added to buffer solution of APF and then ferrous sulfate was added. The fluorescence increases were the fluorescence intensities minus the base-line signal obtained after adding $0.1 \mathrm{mM} \mathrm{H}_{2} \mathrm{O}_{2}$ 


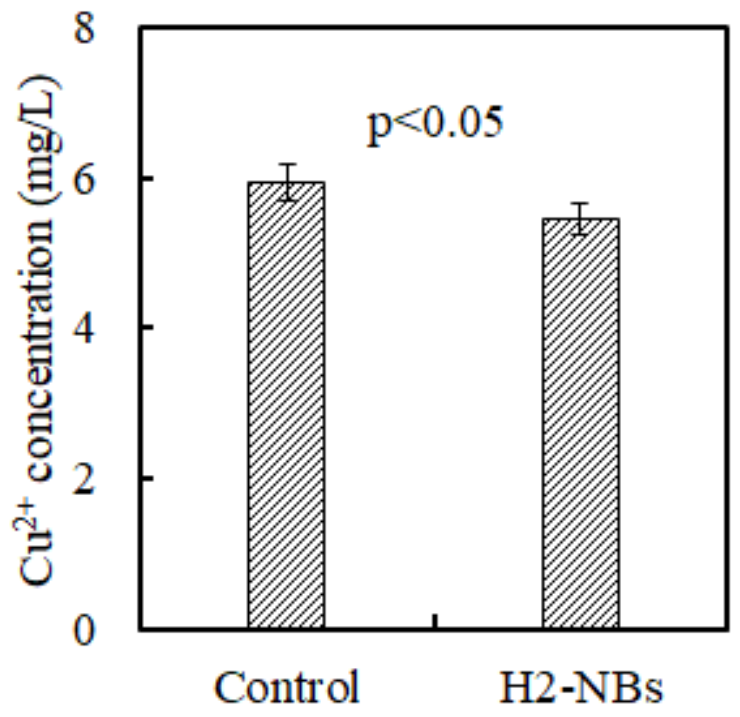

Figure S4 Effect of hydrogen NBs on the concentration of divalent copper ions in water. Error bars are the standard errors of three replicates. 
Table S1 Two-way ANOVA testing for the influence of different treatments and copper concentrations on the ROS of Chlorella.

\begin{tabular}{lclllc}
\hline & \multicolumn{5}{c}{ Reactive Oxygen Species } \\
\cline { 2 - 6 } & df & SS & MS & F & P \\
\hline Treatments & 2 & 0.0760 & 0.0380 & 5.7068 & 0.0222 \\
Concentrations & 5 & 0.1780 & 0.0356 & 5.3475 & 0.0119 \\
\hline
\end{tabular}

SS refers to the Sum of Squares; MS refers to Mean Square; All the F values are based on the residual mean square error; The $\mathrm{P}$-values test the statistical dignificance of each of the factors. 